latter, as far as I am aware, always remains conscious of his own identity. It would seem that the schizophrenic is in this respect much the same as the dreamer. That is to say, hidden by the delusional fantasies is the normal ego which, on occasions, is apt to reassert itself. Every psychiatrist has seen this happen when a patient is visited, in bodily illnesses, or in cases that unexpectedly recover. In these cases it is often found that the patient has an astonishing memory of all that has taken place during the period of his psychosis. It has always seemed to me to be rather curious that the highest and most recently acquired cognitive functions-mental efficiency and memory-should be preserved in these cases. One might think that, in view of educational pressure and the dreadful examinations which young people are called upon to pass, a kind of premature mental enfeeblement might result in many patients. Yet this is not the form taken by adolescent cases, many of which occur in those of brilliant intellects to whom the passing of examinations is mere child's play. What we see in our adolescent patients is not the deterioration of the most recently acquired functions, but rather of the oldest and most essential functions-namely, those of instinct and feeling, the inharmonious development of which is responsible for the symptoms exhibited.

\section{INTELLECT AND INSTINCT}

It has to be recognized that intellect, as distinguished from feeling and emotion, is not an indispensable element of life. Intellect is more or less of a luxury, and a high intelligence is of less importance than a stout heart-that is to say, a vigorous, balanced, and harmonious affective life. In view of the considerable incidence of mental disorders in civilized communities, both within and outside the mental hospitals, it is perhaps justifiable to inquire whether there is not some increase in the incidence of those who are the subjects of some constitutional vitiation of the organic processes concerned with the development of the "springs of action" - that is to say, of the instinctive-emotional life.

\section{PUERPERAL SEPSIS: THE IMPORTANCE OF EARLY TREATMENT} BY

\section{A. REMINGTON HOBBS, M.D., M.R.C.P., M.C.O.G.} MEDICAL SUPERINTENDENT, ST. MARY ABBOTS HOSPITAL, KENSINGTON (L.C.C.) ; CONSULTING GYNAECOLOGIST TO THE ROYAL BOROUGH OF KENSINGTON

Anyone, seriously engaged in the study of puerperal sepsis, who possesses experience of a large number of cases, must realize the value of early treatment. The recognition of this fundamental principle in respect of other diseasesfor example, appendicitis, intestinal obstruction, syphilis, and diphtheria-has been the one and only means of reducing mortality from these diseases.

In the treatment of puerperal sepsis the method of glycerin irrigation, as practised at this hospital, has been proved to be entirely $\cdot$ harmless to living cells, and has established its claim to be regarded as the most efficient remedy at our disposal. Since this is so, why should not this method of treatment be applied at an earlier stage, in the ante-natal period for example, as well as on a larger scale at an earlier period in the puerperium, instead of postponing treatment until the uterine tissues become hopelessly infected and the blood stream invaded? The death rate from puerperal sepsis is not only practically stationary, but is far too high. Many women are left with a legacy of chronic invalidism, and swell the ranks of those who attend gynaecological clinics, when, by early and adequate treatment, any serious complication might have been prevented. Many even go into labour with sepsis already established, in spite of ante-natal clinics, and in spite of our present-day knowledge of bacterial infections and antiseptics.

The following are the reasons, in my opinion, for the present unsatisfactory state:

1. Too much stress has been placed on the fact that the phenomena of the so-called normal puerperium are of a physiological character.

2. There is a widespread belief, unwarranted by the facts, that puerperal sepsis is always characterized by fever, the result of bacterial infection of the raw uterine surface.

3. Treatment is in many cases not begun ear'y enough, nor is it continued until every symptom and sign of sepsis has disappeared, and the uterus has been restored to its normal condition.

4. Most important of all, and as a corollary to No. 2, early symptoms and signs have been too long neglected by medical attendants in maternity cases.

With regard to the first reason, it must be borne in mind that after labour the raw uterine surface is an open wound ; as in the case of other wounds, simple reactionary or inflammatory changes must occur in the course of healing. These reactionary changes are at a minimum in cases where the uterine surface is left perfectly clean, and where it remains clean. After labour, however, placental remnants and blood clot frequently remain attached to the uterine wall, and only gradually are they extruded. So long as they remain in the uterus they are undoubtedly a potential nidus for infecting organisms of varying degrees of virulence.

The line between what may be regarded as physiological and normal on the one hand, and pathological on the other, is too fine to be drawn; the one may merge insensibly into the other. I contend that for every case of severe puerperal sepsis there are hundreds where a mild degree of sepsis exists unrecognized, but which nevertheless leads to untold suffering among women. This brings me to the second of the reasons given above-namely, that there is too widespread a belief that puerperal sepsis is always a febrile disease. Nothing could be further from the truth, and, until we rid our minds of this idea, little progress will be made towards combating the risks of maternity.

In every published list of statistics a temperature of $100.4^{\circ} \mathrm{F}$. is regarded as the sign of puerperal sepsis. There are many instances, some to our sorrow, in which we have wasted valuable time and have exposed our patients to the greatest risk, through too blind an acceptance of the current teaching. Temperature, as an index of early pathological changes in the uterus, is by itself a thoroughly unreliable guide. All the signs of pathological inflammation must be taken into account, and, if we must give pride of place to one, surely it should be to the pulse rate.

A.recent case which came under our notice will illustrate what I have just written. This patient had a normal temperature, but her pulse rate ranged from 104 to 120 . At the end of four days her temperature rose to $101^{\circ}$, and, as the standard of morbidity had been passed, active treatment was begun. The patient, however, died of septicaemia.

In my experience it is exceptional for cases of puerperal sepsis to develop pyrexia at the outset. Many patients have offensive lochia, red lochia, and purulent discharge, and yet for some days remain entirely apyrexial. Further, while severe cases of puerperal inflammation are due to bacterial invasion of the raw uterine surfaces, there is a much greater number where inflammatory changes occur as a result, purely, of the retention of placental fragments 
and blood clot, and, as I have stated, those are the cases that frequently lead to chronic invalidism among women. Unfortunately little or nothing is done at the very time when much could be done, and the most favourable opportunity to deal with them is lost. Following labour or miscarriage, much blood and serum, together with fragments of placenta, normally continue to escape from the uterus for several days. Anything that interferes with the natural drainage will undoubtedly give rise to signs and symptoms; we must learn to recognize these, for they are certainly the earliest heralds of possible trouble later. A full appreciation of their significance will enable us to begin early and efficient treatment, by which alone we can hope to reduce maternal mortality.

As will be seen from the diagram there are various points at which interference with free drainage may occur.

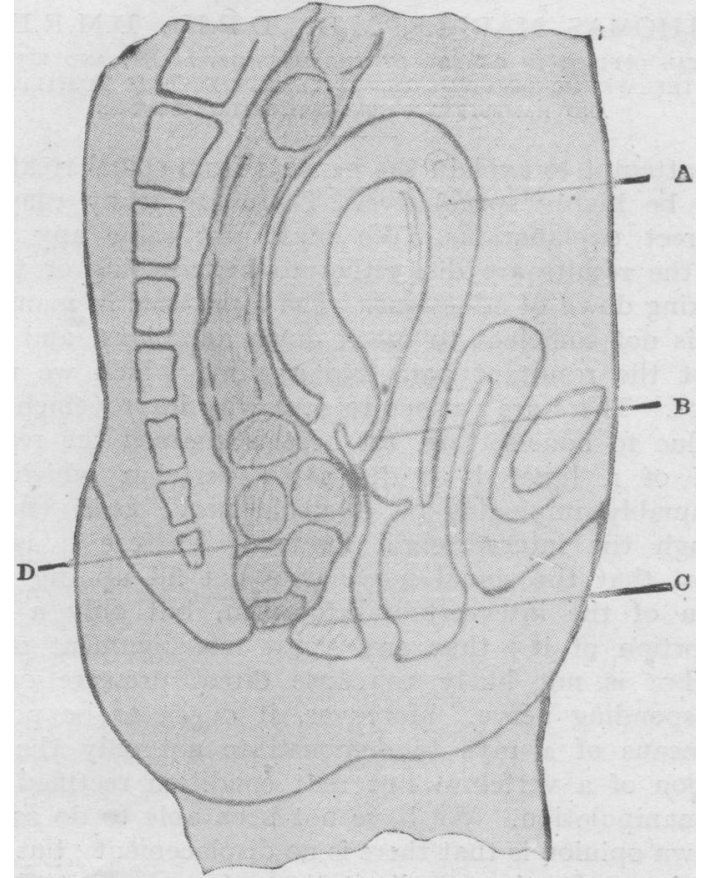

Sketch showing the four main positions at which the uterine secretions may be obstructed. A, Iterine wall.
B, Cervical canal. C, Swollen perineum. D, Loaded rectum.

These may be grouped under two headings: (1) intrauterine ; and (2) extrauterine.

In the first group the interference may occur in the uterus itself. This may be the result of an unhealthy condition of the uterine wall antedating conception, or it may be caused by trauma produced by the finger, forceps, curette, styptics, or hot intrauterine douches. Interference with free drainage may also occur owing to obstruction of the cervical canal by fragments of blood clot and placental tissue, or by a swollen oedematous condition of its wall. Cervical obstruction is most likely to occur in the early stages of the puerperium, and calls for immediate inspection. If conditions are such that thorough examination is impracticable in the patient's home, she ought to be sent to hospital without delay, where active treatment may be begun.

Another matter that has some bearing, not only on the maintenance of free drainage during the puerperium, but also on the subsequent health of the uterus, concerns the position of that viscus. At the end of the first week of the puerperium, when the uterus is again returning to the pelvis, there is a danger of retroversion ; it is important, therefore, to correct this tendency by placing the patient in the prone position at frequent intervals, in addition to adopting the semi-Fowler position. Among the extrauterine causes of interference with f:ee arainage may be mentioned the following: a loaded rectum, an over-distended bladder, and oedematous vaginal walls in association with pelvic cellulitis or oedema of the perineum. The first is sufficiently obvious, and is easily remedied, but a note of warning is necessary here-the patient's symptoms may be due, not to the toxic absorption from a loaded bowel, but to interference with free escape of discharges from the uterus; in such cases special attention should be paid to measures calculated to improve subsequent free drainage. I have discussed this question, however, in greater detail in a paper read before the Royal Society of Medicine in 1924.

I have stated above that in my opinion active treatment is not begun soon enough, nor is it continued as a rule until the uterus has been restored to a perfectly healthy condition. The truth of this has been borne in upon me as the result of having had to deal with many cases admitted to hospital after the usual expectant or conservative treatment had been tried, and had been found wanting.

In cases where thorough glycerin treatment has been commenced early enough I have met with no instance where this method has failed to relieve uterine obstruction and to induce free and efficient drainage. Not only so, but during the last eighteen months, treatment at this hospital has been more thorough and prolonged, and has not ceased until the uterus has been restored to a perfectly normally functioning organ, and until all cervical erosions have been completely healed. By way of illustration I would like to mention a severe case which was recently admitted on the eighth day of the puerperium. The cervix was lacerated and eroded, and there was extensive pelvic cellulitis. The patient was treated on 135 occasions, $29,450 \mathrm{c} . \mathrm{cm}$. of glycerin being given. After forty-six days the cervix had entirely healed. The detailed technique has been fully described in several previous papers.

Lastly, I would enter a plea for the recognition of the earliest symptoms and signs of puerperal disease. These have been too much neglected. It is becoming increasingly evident that every stage from pregnancy to the labour room and during the post-natal period, must be more closely watched. Actually, many women go into labour with sepsis already established. In order to minimize the risks, cervical erosions should be noted early in pregnancy, and steps be taken to treat the condition. Treatment should be carried out by regular instillations of glycerin, one inch within the cervix, throughout pregnancy, in order to reduce inflammation as much as possible. The presence of $B$. coli in the urine requires treatment in the cervical canal, and when a coliform infection of the urine exists, a similar infection will frequently be found in the lower genital tract. The treatment of both uterus and bladder, where such an infection has been present, should be actively continued during the puerperium. There is a further field forglycerin treatment in the toxaemias of pregnancy, where, after a fair trial, domiciliary treatment has failed and the patient has reached the limit of intoxication.

Cases which demand treatment fall into two groups:

(1) Those patients who suffer from headaches, drowsiness, oedema, increasing blood pressure and jaundice, and whose urine contains tube casts and albumin ; and

(2) Those who suffer from haemorrhage.

These patients should be sent into hospital early for efficient treatment. Failure to do so permits progressive damage to occur in the organs, such as the kidneys and liver, and may endanger the patient's life. Experience of years has taught us that the lying-in ward is a surgical ward, and that it contains many cases which deviate from the normal. 
What are the chief signs and symptoms which, if detected early, reduce the mortality and morbidity? They are:

(1) A temperature of $99^{\circ}$, and a pulse rate of 90 ;

(2) Uterine colic ; and

(3) Abnormal lochia.

With regard to the first, valuable time may be lost if the textbook standard of morbidity-namely, a temperature of $100.4^{\circ}$-is awaited. It is our practice to institute drainage by glycerin as soon as ever the temperature rises to $99^{\circ}$, or the pulse rate to 90 .

Given an empty uterus, devoid of inflammation, and in good position, the patient will be free from pain. Should pain be present, a cause for it can always be found if sought for ; almost invariably that cause will be interference with free drainage. Pain is the cardinal symptom, and tenderness is the cardinal sign of an obstructed viscus, yet many medical practitioners still labour under the delusion that it is natural for a woman to suffer from after-pains for the first few days of the puerperium. These after-pains are really symptoms of a slight interference with the free escape of fluids from the uterus, and, as such, are a danger signal. If not relieved within twe!ve hours, active steps should be taken to encourage free drainage ; otherwise, the case may end in disaster.

The presence of offensive lochia is another indication for early treatment, especially since many of these cases do not in the early days show pyrexia. Profuse lochial discharge means, as a rule, inflammation of the uterine wall, and accordingly demands early treatment. As a general rule, it may be laid down that the red lochia should cease in a healthy woman about the sixth or seventh day, and those cases which do not conform to this rule should receive hospital treatment until all signs of inflammation have disappeared.

Early and efficient treatment along the lines that I have indicated would undoubtedly save many lives, and would lessen the number of those who frequent gynaecological out-patient clinics. It would obviate many useless curettings, and hysterectomy would be less frequent.

To illustrate the type of case in which glycerin treatment has been found valuable, I append the following figures taken from a recent six-month period. They relate to a total of $\mathbf{2 0 8}$ deliveries occurring in this hospital.

\begin{tabular}{|c|c|c|c|c|}
\hline & & & $\underset{\text { Cases }}{\text { Number of }}$ & $\begin{array}{l}\text { Average Number } \\
\text { of Treatments } \\
\text { per Patient }\end{array}$ \\
\hline 1. Cervical erosion... $\quad \ldots$ & $\cdots$ & $\ldots$ & 107 & 112 \\
\hline 2. Secondary albuminuria & ... & $\ldots$ & 10 & 46 \\
\hline 3. Bacillus coli infection .. & $\cdots$ & $\ldots$ & 60 & 75 \\
\hline 4. Ante-partum haemorrinag ${ }$ & $\cdots$ & $\cdots$ & 6 & 36 \\
\hline 5. Placenta praevia & $\cdots$ & $\cdots$ & 3 & 10 \\
\hline 6. Inteina1 manipulation & $\cdots$ & $\cdots$ & 3 & 6 \\
\hline 7. Fo:ceps deliveries & $\cdots$ & $\ldots$ & 10 & 8 \\
\hline 8. Retained placenta & $\cdots$ & $\cdots$ & 6 & 36 \\
\hline 9. Post-partum haenourhage & $\cdots$ & $\cdots$ & 8 & 14 \\
\hline 10. Retained memb:ane.. & $\cdots$ & $\cdots$ & 12 & 18 \\
\hline 11. Uterine colic $\quad \ldots$ & ... & $\cdots$ & 31 & 32 \\
\hline 12. "Three nines"*... & $\cdots$ & $\cdots$ & 45 & 24 \\
\hline 13. Offensive lochia ... $\ldots$ & $\cdots$ & $\cdots$ & 20 & 32 \\
\hline 14. Sscondary haemorrhage & $\cdots$ & $\cdots$ & $\vdots 0$ & 18 \\
\hline 15. Pu $\in$ rperal thrombo-phlebiti & & $\cdots$ & 8 & 64 \\
\hline
\end{tabular}

*Increased temperature $\left(99^{\circ}\right.$ or over), increased pulse rate ( 30 or over).

The majority of the $20 \mathrm{~S}$ patients suffered from more than one of the above pathological conditions. The amount of glycerin used in each case varied from a few cubic centimetres to 200 c.cim., injected once or three times daily either into the uterine cavity or cervical canal.

A recent list from the gynaecological wards over a three-month period showed that 33 patients were discharged with healed cervical erosions, the average number of g!ycerin treatments being 26 , and the average number of days in hospital being 19 .

I wish to thank Sir Almroth Wright, Dr. Leonard Colebrook, and Professor Robert Donaldson for their great help in the study of this disease, also Drs. Harold Carter, Joyce E. M. White, and Margaret E. Anderson, for assisting in the treatment.

\section{SOME COMMON COMPLAINTS AMENABLE TO SPINAL TREATMENT}

BY

THOMAS MARLIN, M.D., D.P.H., D.M.R.E. MEDICAL OFFICER IN CHARGE OF THE MASSAGE, LIGHT, ANI ELECTROTHERAPECTIC DEPARTAENTS, UNIVERSITY COLLEGE HOSPITAL AND HAMPSTEAD GENERAL HOSPITAL, LONDON

Any attempt to explain the benefits from spinal treatment must be highly speculative. There are many easy but incorrect explanations. We must put aside any claim that the results are due either to bone-setting or to the breaking down of adhesions. The force used in manipulation is not sufficient to break down adhesions, and there is not the resultant pain and swelling which we would expect. There are various reasons why improvements are not due to bone-setting, for here is claimed the replacement of a luxated or dislocated vertebra which was presumably impinging on a spinal nerve as it emerged through the intervertebral foramen, whereas it is well known that the spinal nerve does not fill up the whole lumen of the intervertebral foramen, but only a small proportion of it ; thus any slight mis-alignment of one vertebra is not likely to cause direct pressure on the corrasponding nerve. Moreover, it ought to be possible by means of $x$ rays to demonstrate not only the malposition of a vertebra, but that condition rectified after the manipulation. We have not been able to do so, and our own opinion is that there is no displacement ; but there may be a fixation or limitation of movement due to some static cause, and any sound heard during a spinal manipulation, as usually carried out, is due merely to a separation of the articular surfaces, and not to the replacement of anything that has been out of place. Indeed, in Case I (under Dr. McNee) $x$-ray examination was made before and immediately after the manipulation. The second series of photographs showed that the tilting had apparently been overcome. At one or two subsequent examinations the good position seemed to be maintained, but the radiologist, by making. a slight change in the position of the patient, was able to reproduce in a photograph the exact tilting of the fourth lumbar vertebra which was seen at the first examination.

- Luck may be a factor--the luck of some new form of treatment producing a favourable impression on the patient who returns at subsequent sittings prepared for better and better results. This factor, however, is not unknown in other methods of treatment, where a psychological stimulus is used to influence physiological processes. But with the spinal treatment we are able to break into the abnormal chain of circumstances, using a physical stimulus at a definite part. When we have on the one hand some ailment or disability, and on the other hand contracted tissue giving rise to pain on pressure round particular parts of the spine, whether as a reflex from the former or whether the former is a reflex from the latter, if we are able to break in on that reflex arc, perhaps we can upset the whole vicious cycle 